\title{
Mechanical interaction between cells and fluid for bone tissue engineering scaffold: Modulation of the interfacial shear stress
}

\author{
L.D. Blecha ${ }^{\text {a }}$, L. Rakotomanana ${ }^{\text {b }}$, F. Razafimahery ${ }^{\text {b }}$, A. Terrier ${ }^{\text {a }}$, D.P. Pioletti ${ }^{\text {a,* }}$ \\ ${ }^{a}$ Laboratory of Biomechanical Orthopedics, Ecole Polytechnique Fédérale Lausanne, EPFL/STI/IBI/LBO, Station 15, 1015, Switzerland \\ b IRMAR, University of Rennes I, 35042 Rennes Cedex, France
}

\section{A R T I C L E I N F O}

Article history:

Accepted 1 November 2009

Keywords:

Cell fluid interaction

Shear stress

Analytical solution

Bone tissue engineering

\begin{abstract}
A B S T R A C T
An analytical model of the fluid/cell mechanical interaction was developed. The interfacial shear stress, due to the coupling between the fluid and the cell deformation, was characterized by a new dimensionless number $N_{f s}$. For $N_{f s}$ above a critical value, the fluid/cell interaction had a damping effect on the interfacial shear stress. Conversely, for $N_{f s}$ below this critical value, interfacial shear stress was amplified. As illustration, the role of the dynamic fluid/cell mechanical coupling was studied in a specific biological situation involving cells seeded in a bone scaffold. For the particular bone scaffold chosen, the dimensionless number $N_{f s}$ was higher than the critical value. In this case, the dynamic shear stress at the fluid/cell interface is damped for increasing excitation frequency. Interestingly, this damping effect is correlated to the pore diameter of the scaffold, furnishing thus target values in the design of the scaffold. Correspondingly, an efficient cell stimulation might be achieved with a scaffold of pore size larger than $300 \mu \mathrm{m}$ as no dynamic damping effect is likely to take place. The analytical model proposed in this study, while being a simplification of a fluid/cell mechanical interaction, brings complementary insights to numerical studies by analyzing the effect of different physical parameters.
\end{abstract} (c) 2009 Elsevier Ltd. All rights reserved.

\section{Introduction}

When a fluid flows on a structure, a shear stress is induced at the interface between the fluid and the structure. This interfacial shear stress has been supposed to play a key role in mechanobiology. Indeed, a large number of experimental studies succeed in correlating the magnitude of the interfacial shear stress with cells behavior such as for metabolite production (Frangos et al., 1988) and atheroprotective gene expression profile (Malek et al., 1999) in endothelial cells, alkaline phosphatase activity (Goldstein et al., 2001) and intracellular cyclic adenosine monophosphate (Reich et al., 1990) in osteoblastic cells, or nitric oxyde and prostaglandin $\mathrm{E}_{2}$ release in fibroblasts (Pauw et al., 2000).

Fluid flow is then an important parameter to consider in the development of artificial bone scaffold to favor osteointegration and osteoinduction. In addition to ensuring the transport of nutriment and waste, the fluid motion inside the scaffold also exerts shear stresses on cells seeded in the scaffold that are shown to increase the production and release of TGF- $\beta_{1}$ and other growth factors (Kapur et al., 2003 ; Lau et al., 2006 ; Sakai et al., 1998). These proteins are essential for the recruitment inside the scaffold of mesenchymal stem cells and for their differentiation into bone

\footnotetext{
* Corresponding author: Tel.: +4121693 83 41; fax: +41216938660.

E-mail address: dominique.pioletti@epfl.ch (D.P. Pioletti).
}

forming cells (Huang et al., 2002; Panagakos, 1994). An artificial bone scaffold that induces an adequate fluid motion due to mechanical loading is thus likely to enhance its osteointegration and osteoinduction properties. Following these observations, we recently presented an optimization method allowing to determine mechanical and morphological parameters for an artificial scaffold favoring bone formation through mechano-transduction aspects (Blecha et al., 2009).

On a smaller length scale, the local changes of fluid velocity near the cell membrane may modulate the shear stress. Although, the steady interfacial shear stress is a good measure of the effective mechanical stress exerted by a periodic fluid flow at low frequency, it may be misleading in general. Indeed, the shear stress induced by the fluid flow causes the cell to deform which in turn affects the fluid flow. A coupling phenomenon known as fluid/structure interaction is taking place. As a result of this coupling, the fluid flow and the shear stress at the interface between the fluid and the cells may be very different than the ones of a steady-state condition.

While fluid/structure interaction is widely studied in engineering fields such as aeronautics, it seems that biomechanics has not well considered its impact on the mechanical description of cells stimulated by fluid flow. In the particular case of artificial bone scaffold, it would be interesting to analyze cell mechanical stimulations considering the effects of fluid-structure interactions. 
The resolution of a fluid-structure interaction problem is usually performed numerically and does not allow to fully catch the global physics involved in this complex situation. To this end, an analytical resolution of a simplified time-dependent model for the cell/fluid interaction could bring a better insight on the importance of this interaction and hopefully could bring new ideas to be tested experimentally.

In this study and thanks to the analytical approach followed, a novel dimensionless number $N_{f s}$ that we will call the fluid/cell interaction number is defined. Based on the fluid and solid physical and geometrical characteristics, this fluid/cell interaction number characterizes the coupling phenomenon between the fluid and the cells. Its magnitude was found to be an indicator for the magnitude of the shear stress amplification or damping due to dynamic phenomena, which furnishes valuable information to characterize the cells behaviors in clinical relevant situations. In particular, its application to bone scaffold colonized by a layer of cells might help to define a specific pore diameter range that favors cell stimulation.

\section{Analytical model}

\subsection{Configuration}

A Newtonian incompressible fluid of dynamic viscosity $\eta$ and mass density $\rho_{f}$ was confined between two infinite plates separated by a distance $H$ (Fig. 1 ). One plate was a semi-infinite elastic solid of height $h$, which represented a monolayer of cells fixed to a rigid wall. The other plate was rigid and was oscillating in the tangential direction at an harmonic velocity $v_{0} \sin \omega_{0} t$ representing the oscillating fluid flow. The cells, the plates and the fluid were initially at rest.

\subsection{Governing equations and assumptions}

The fluid/cell interaction model was derived from continuum mechanics, together with four assumptions given below. First, the interaction was considered in a one dimension, with time dependency, i.e. the fluid velocity $v$ and pressure $p$ as well as the solid displacement $u$ were assumed to be function of the cross

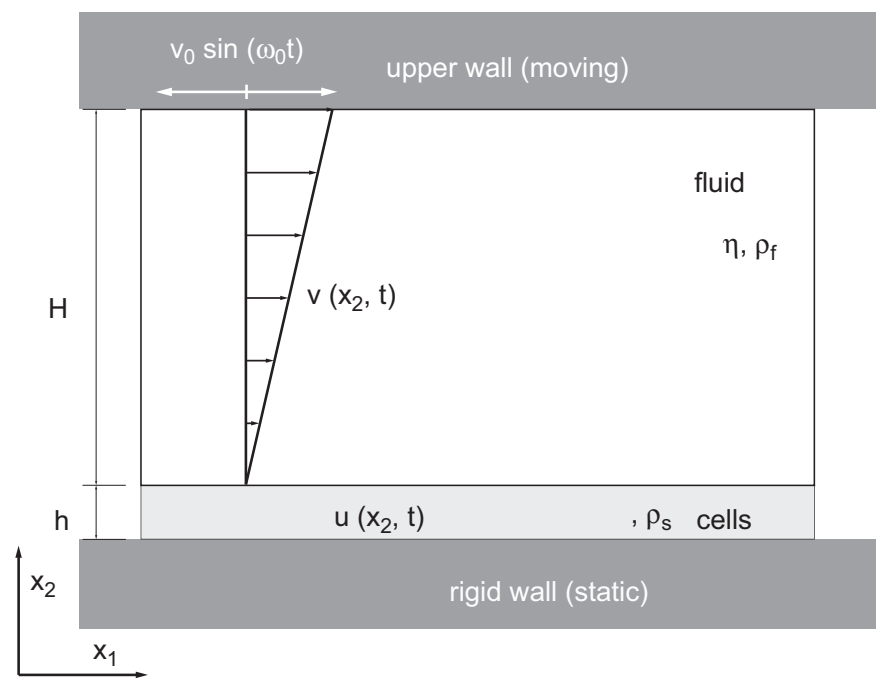

Fig. 1. Simplified configuration of a cell layer stimulated by an oscillating fluid flow. flow direction $x_{2}$ and time $t$ :

$v=v\left(x_{2}, t\right) \quad p=p\left(x_{2}, t\right) \quad u\left(x_{2}, t\right)$

\subsubsection{Fluid}

The motion of the Newtonian incompressible fluids described by the mass continuity equation together with the Navier-Stokes equations were rewritten after neglecting all external volume forces and defining the dimensionless (stared *) variables

$v=v^{*} V, \quad x=x^{*} L, \quad t=t^{*} T, \quad p=p^{*} \frac{\eta V}{L}$

to the dimensionless form

$\operatorname{Re} \cdot S t \frac{\partial v^{*}}{\partial t^{*}}+\operatorname{Re}\left(v^{*} \cdot \nabla^{*}\right) v^{*}=-\nabla^{*} p^{*}+\Delta^{*} v^{*}$,

$R e$ is the Reynold's number and $S t$ is the Strouhal number. As a large category of biological fluid flows are taking place at very low Reynold's and Strouhal's numbers

$R e \leq 1$ and $R e \cdot S t \leq 1$,

the convective and inertial terms of the Navier-Stokes equations (3) were neglected. However, the assumption $R e \cdot S t \leq 1$ restricted the domain of validity of the fluid description. In the particular case of this study, the excitation frequency was therefore restricted to

$f<f_{\text {crit }}$ where $f_{\text {crit }}=\frac{\eta}{\rho_{f} H^{2}}$.

Finally, the fluid equations were

$\nabla \cdot v=0$

$\eta \Delta v=\nabla p$.

The non-slip condition in $x_{2}$-direction and the no-flow (impermeable) condition in $x_{1}$-direction were assumed at $x_{2}=H+h$ :

$v_{1}(H+h, t)=v_{0} \sin \left(\omega_{0} t\right) \quad v_{2}(H+h, t)=0 \quad \forall t>0$

And, the system was assumed at rest for $t=0$ which gave the following initial conditions:

$v_{1}\left(x_{2}, 0\right)=v_{2}\left(x_{2}, 0\right)=p\left(x_{2}, 0\right)=0 \quad \forall x_{2} \in[h, h+H]$

\subsubsection{Cell layer}

The equilibrium of forces in a linear elastic homogeneous isotropic cell layer was described by the Navier equations, which were expressed in terms of the deformation field $u$

$(\lambda+\mu) \nabla(\nabla \cdot u)+\mu \Delta u+f=\rho_{s} \frac{\partial^{2} u}{\partial t^{2}}$,

where $\lambda$ and $\mu$ are the Lamé coefficients. Similarly to the fluid domain, the volume forces were assumed zero in the solid domain, i.e. $f=0$. Finally, the lower edge of the cell layer was fixed in all translations to a rigid and static wall

$u_{1}(0, t)=0 \quad u_{2}(0, t)=0 \quad \forall t>0$

and the cell layer was considered at rest for $t=0$

$u_{1}\left(x_{2}, 0\right)=0 \quad u_{2}\left(x_{2}, 0\right)=0 \quad \forall x_{2} \in[0, h]$.

\subsubsection{Fluid-cell interaction}

The continuity of forces and velocities at the interface $\Gamma_{f_{s}}$ between the two media created the coupling between the cell layer and the fluid. The kinetic coupling between the elastic layer and the fluid was

$v=\frac{\partial u}{\partial t} \quad \forall x_{2} \in \Gamma_{f s} \quad$ and $\quad t>0$. 
The dynamic coupling was given by the continuity of the stress at the interface

$\sigma_{f} n_{f}=\sigma_{s} n_{s} \quad \forall x_{2} \in \Gamma_{f s}$ and $t>0$.

where $\sigma_{f}$ and $\sigma_{s}$ are respectively the fluid and the cell stress. The unit normal vector at the fluid-solid interface was denoted by $n_{f}$ resp. $n_{s}$. The constitutive law of the Newtonian incompressible fluid was (Chevray and Mathieu, 1993)

$\sigma_{f}=-p I+\eta\left(\nabla v+\nabla v^{T}\right)$

and the constitutive equation for the linear elastic medium was written in terms of solid displacement $u$

$\sigma_{s}=\lambda(\nabla \cdot u) I+\mu\left(\nabla u+\nabla u^{T}\right)$.

\subsection{Analytical solution}

The assumptions applied on the solid (10) and fluid Eqs. (6)-(7) yielded to a one dimensional time-dependent fluid/cell interaction problem. The initial and boundary conditions (8)-(9),(11)-(14) allowed expressing the solid displacement $u$, fluid velocity $v$ and pressure $p$ as functions of $x_{2}, t$ and of the unknown shear stress at the interface $\tau(t)$. After different analytical manipulations (see Appendix 1 for the principal steps and Supplementary Website Material for a complete derivation of the equations), it was possible to find an analytical solution for the interfacial shear stress $\tau(t)$ :

$\tau(t)=\tau \sin \left(\omega_{0} t+\delta\right)$

where $\tau$ is the interfacial shear stress magnitude. Eq. (17) shows that the interfacial shear stress oscillates at the same frequency as the excitation, but delayed of a phase shift $\delta$. We define the normalized interfacial shear stress $\tau^{*}$ by normalizing the interfacial shear stress magnitude $\tau$ with the corresponding steady interfacial shear stress magnitude $\tau_{0}=v_{0} \eta / H$ (see Supplementary Website Material for complete derivation)

$\tau^{*}=\frac{\pi^{2} \mu H}{\left(\pi^{2}-8\right) h \eta} \frac{\omega_{0}^{2}+\omega_{1}^{2}}{\sqrt{\omega_{0}^{6}+J_{2} \omega_{0}^{4}+J_{1} \omega_{0}^{2}+J_{0}}}$

with

$$
\begin{gathered}
J_{2}=\frac{\pi^{4}}{2\left(\pi^{2}-8\right)^{2}} \frac{\mu}{h^{2} \eta^{2} \rho_{s}}\left[2 H^{2} \mu \rho_{s}-\eta^{2}\left(\pi^{2}-8\right)\right] \\
J_{1}=-\frac{\pi^{6}}{16\left(\pi^{2}-8\right)^{2}} \frac{\mu^{2}}{h^{4} \eta^{2} \rho_{s}^{2}}\left(8 H^{2} \mu \rho_{s}-\eta^{2} \pi^{2}\right) \\
J_{0}=\left[\frac{\pi^{4} H \mu^{2}}{4\left(\pi^{2}-8\right) h^{3} \rho_{s} \eta}\right]^{2}
\end{gathered}
$$

Analysis of Eqs. (18)-(19) showed first that $\tau^{*}$ is always positive for all $\omega_{0}, \eta, \mu, \rho_{s}, h, H$ greater than zero. Second, the normalized interfacial shear stress $\tau^{*}$ takes the unit value for $\omega_{0}=0$ and tends to zero for $\omega_{0} \rightarrow \infty$. Third, no singularities are present in the range of positive excitation frequency. Finally, taking the first derivative of $\tau^{*}$ with respect to the excitation frequency $\omega_{0}$ showed that the normalized interfacial shear stress has one or two extrema, depending on the value of the dimensionless number $N_{f s}$ defined as

$N_{f s}=\frac{\eta}{H \sqrt{\mu \rho_{s}}}$.

The dimensionless number $N_{f s}$, that we called fluid-cell interaction number, came out after analysis of the discriminant of the sixth order polynomial appearing at nominator after derivation of (18). For values of $N_{f s}$ smaller than $4 / \pi$, the normalize interfacial shear stress $\tau^{*}$ has one and only one extremum, which is a maximum (Fig. 2a). In other words, the
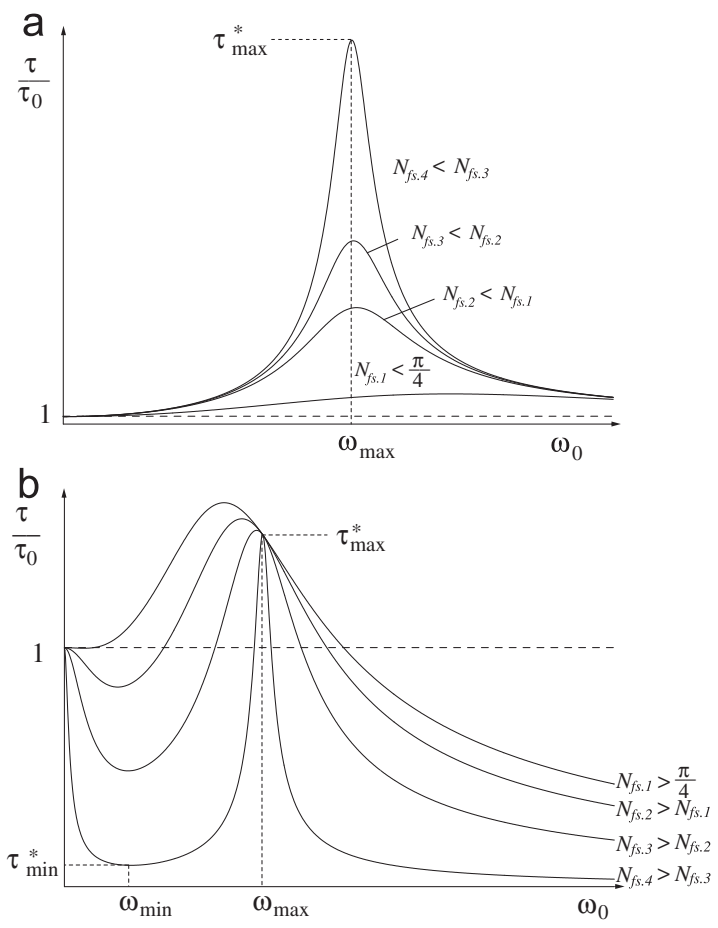

Fig. 2. Dimensionless shear stress at the interface between fluid and cell layer in function of the excitation frequency for (a) $N_{f_{s}}<4 / \pi$ and (b) $N_{f s}>4 / \pi$. The magnitude of $N_{f s}$ is related to the normalized interfacial shear stress.

normalized interfacial shear stress $\tau^{*}$ follows a resonance-like behavior. The location and magnitude of the maximum depend on three parameters which are $H / \eta, \mu$ and $\rho_{s}$, and are independent of the cell height $h$ and fluid density $\rho_{f}$.

For values of $N_{f s}$ higher than $4 / \pi$, the normalize shear stress has two extrema-a minimum and a maximum. The normalized interfacial shear stress first decreases rapidly towards a local minimum. Then, it increases towards a maximum upper bounded by 1.48 and finally tends to zero for $\omega_{0} \rightarrow \infty$ (Fig. 2b). The magnitude and location of the local minimum depend on three parameters which are again, $H / \eta, \mu$ and $\rho_{s}$, and are also independent of the cell height $h$ and fluid density $\rho_{f}$.

\section{Results}

\subsection{Application to bone scaffold}

The role of dynamic fluid/cell mechanical coupling was studied in a specific biological situation involving cells seeded in a bone scaffold. The typical configuration of bone cells inside a PLLA scaffold pore (Mathieu et al., 2006) was estimated from $\mu$-CT images of dry constructs (Scanco Medical AG, Bassersdorf, Switzerland). The averaged pore diameter was computed from these images with help of a dedicated algorithm (Hildebrand and Ruegsegger, 1997). The cell layer that colonizes the pore walls was assumed to have a height $\mathrm{h}$ of $20 \mu \mathrm{m}$. The fluid and cell layer mass densities were assumed to be similar to water density. Finally, the cell shear modulus was taken from the literature (Caille et al., 2002). All values used in the model are summarized in Table 1.

The viscosity of the fluid that stimulates the cells may spread over three to four orders of magnitude. Immediately after implantation, the viscosity of the fluid saturating the pores is of the order of $0.001 \mathrm{~Pa} \mathrm{~s}$, as culture medium is usually used for the 
Table 1

A typical configuration of cell layer inside a bone scaffold.

\begin{tabular}{lcl}
\hline \multicolumn{1}{c}{ Parameter } & Symbol & \multicolumn{1}{c}{ Value } \\
\hline Mean pore size & $\mathrm{H}$ & $480 \pm 220 \mu \mathrm{m}$ \\
Mean cell height & $\mathrm{h}$ & $20 \mu \mathrm{m}$ \\
Cell density & $\rho_{s}$ & $1000 \mathrm{Kg} / \mathrm{m}^{3}$ \\
Mean cell shear modulus & $\mu$ & $500 \mathrm{~Pa}$ \\
Fluid dynamic viscosity (bone marrow) & $\eta$ & $0.1-1 \mathrm{~Pa} \mathrm{~s}$ \\
\hline
\end{tabular}

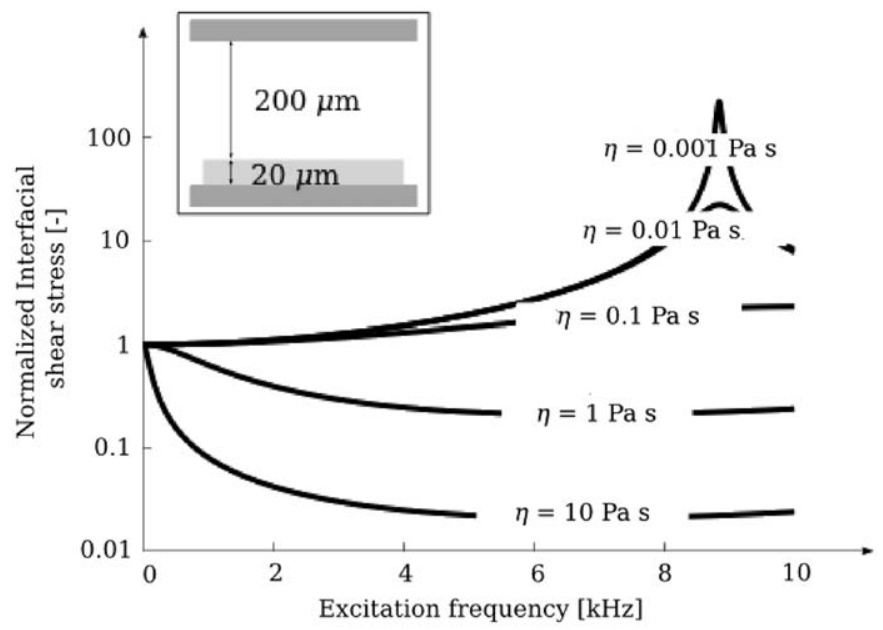

Fig. 3. Normalized interfacial shear stress amplification due to the interaction between a cell layer inside a bone scaffold's pore and a fluid of different viscosity $\eta$. as a function of the excitation frequency.

seeding of cells in the scaffold. Then, the fluid viscosity is likely to increase with time as the initial fluid is gradually replaced by the peri-implant fluid that has a viscosity similar to bone marrow (0.01-1 Pa s (Bryant et al., 1989; Luppe et al., 2002)). Consequently, the dimensionless number $N_{f s}$ is likely to vary with time.

In particular, a scaffold corresponding to the description of Table 1 would exhibit a dimensionless number $N_{f s}$ higher than $4 / \pi$ for fluid viscosity greater than $0.22 \mathrm{~Pa}$ s. In this case, the dynamic shear stress at the fluid/cell interface is damped for increasing excitation frequency (Fig. 2). For instance, the shear stress damping due to a viscous fluid of $1 \mathrm{~Pa} \cdot \mathrm{s}$ was computed to be $1 \%$ at $50 \mathrm{~Hz}$ and to reach $32 \%$ at $1 \mathrm{kHz}$.

On the other hand, a low viscous fluid inside the same artificial bone scaffold was computed to amplify the interfacial shear stress. For instance, a culture medium that has a viscosity of $0.001 \mathrm{~Pa} s$ was computed to induce a maximal amplification of 111 times the steady shear stress at resonance frequency of $8.8 \mathrm{kHz}$ (Fig. 3). However, the shear stress amplification was computed to be less than $2.6 \%$ for excitation frequencies less than $1 \mathrm{kHz}$. Consequently, the shear stress amplification due to the dynamic coupling of a low viscous fluid and cells is likely to be small in the range of frequencies to which a person may be subject.

Indeed, a frequency range between 0 and $1 \mathrm{kHz}$ is often considered to encompass most daily and manual activities such as car driving (1-60 Hz) (Paddan and Griffin, 1998), pneumatic drill or tools in assembly lines $(1-200 \mathrm{~Hz}$ ) (Joshi et al., 2006) or at the extreme of the spectrum chainsaw-induced vibrations $(0-1 \mathrm{kHz})$ (Aatola, 1989). These activities can generate a steady-state mechanical stimulation in the body. In case of transient mechanical stimulation following a shock or an impact, it is common that very high frequencies are generated such as for example following

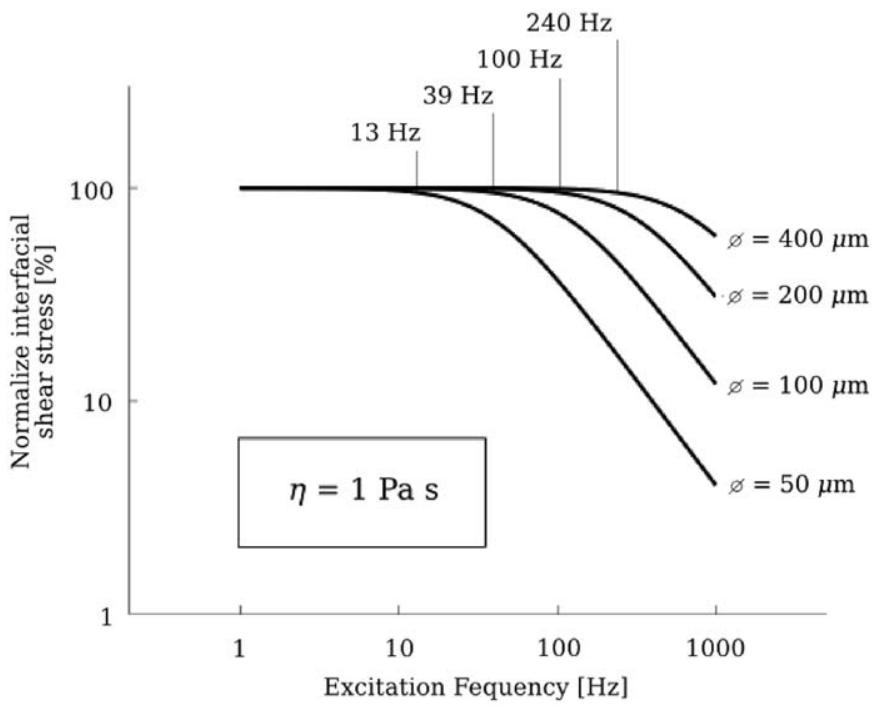

Fig. 4. Impact of the bone scaffold pore diameter on the normalized interfacial shear stress between a cell layer and bone marrow $(\eta=1$ Pa $s)$ as a function of the excitation frequency.

the initial ground reaction force during gait, which can reach $400 \mathrm{~Hz}$ (Gillespie and Dickey, 2003). The corresponding transient stress wave can travel up throughout of the skeletal structure of the body (Whittle, 1999) and affects then the bone scaffold. In this range of frequencies $(0-1 \mathrm{kHz})$, the scaffold pore diameter could play a central role in the modulation of the cell stimulation. Although the fluid/cell interaction had negligible effect on the modulation of the interfacial shear stress for $N_{f s}$ values less than $4 / \pi$, it was computed to have an important damping effect when $N_{f s}$ was greater than $4 / \pi$. In this particular case, the mechanical stimulus on the cells was found to be increasingly damped at frequencies greater than $13 \mathrm{~Hz}$ in a scaffold with $50 \mu \mathrm{m}$ pore diameter (Fig. 4). The normalized interfacial shear stress at the fluid/cell interface was computed to be $63 \%$ of the steady-state shear stress when stimulated at $100 \mathrm{~Hz}$ and was only $4 \%$ at $1 \mathrm{kHz}$. However, the damping magnitude decreased rapidly with increasing pore diameter and was negligible in the frequency range of $0-1 \mathrm{kHz}$ when the scaffold pore diameter was larger than $300 \mu \mathrm{m}$ (Fig. 4). This suggests that a more efficient cell stimulation might be achieved with a construct of pore size larger than $300 \mu \mathrm{m}$ as no dynamic damping effect is likely to take place.

\section{Discussion}

This study focused on the dynamic phenomenon that might modulate cell stimulation embedded in a bone scaffold. The theoretical approach identified a dimensionless number $N_{f_{s}}$ that indicated whether the interaction amplifies or damps the dynamic shear stress at the fluid/cell interface. Pore diameter was found to impact the dynamic coupling phenomenon between the cells attached to the pore surface and the surrounding fluid.

Several studies show that scaffolds with pore diameters larger than $300 \mu \mathrm{m}$ are likely to induce a better and faster osteointegration than scaffolds with smaller pore size (Chang et al., 2000 ; Karageorgiou and Kaplan, 2005 ; Lu et al., 1999). Besides purely biological reasons, the fluid/cell coupling phenomenon could also contribute to explain this empirical result.

It has been shown that high-frequency mechanical stimulation induces higher bone cell response (Qin et al., 1998; Tanaka et al., 
2003) and bone formation (Rubin et al., 2004; Stewart et al., 2005) than steady stimulation. The damping of high-frequency stimulation could perhaps significantly decrease bone cell stimulation. Based on the theoretical development discussed in this study, dynamic damping of high-frequency stimulation was computed for scaffolds with pore diameter smaller than $100 \mu \mathrm{m}$. On the other hand, it was found that scaffolds with pore size larger than $300 \mu \mathrm{m}$ are unlikely to damp dynamic stimulation with excitation frequency below $170 \mathrm{~Hz}$. It may thus be hypothesized that scaffolds with pore diameter larger than $300 \mu \mathrm{m}$ could stimulate more efficiently cells at high frequency than constructs with smaller pore size. Although part of a more complex phenomenon, the mechanical coupling between a cell layer and its surrounding fluid may contribute to explain why scaffold with small pore size are less osteoinductive than constructs with pore sizes larger than $300 \mu \mathrm{m}$. The new dimensionless number $N_{f s}$ is a useful and simple indicator of the type of coupling phenomenon that is likely to take place at the fluid/cell interface and of its magnitude.

The model was intentionally kept simple in many aspects. The objective was to highlight the sensitivity to some mechanical parameters. This choice was made in order to obtain an analytical solution and therefore to develop a global understanding of the problem. Considering the fluid/cell interaction in a one dimensional and time-dependent framework disabled to study the effect of the cell height variation and any phenomena taking place in the other dimensions such as travelling wave flutter observed on dolphin skin (Carpenter et al., 2000). In addition, the assumptions made on the cell's material neglected the viscous effect which may be important in soft tissues (Pioletti and Rakotomanana, 2000) and in cells dynamic responce (Fabry et al., 2001). Nevertheless, this study brought new insights to better understand some basic aspects of the fluid/cell interaction phenomenon that may be part of more complex phenomena.

\section{Conflict of interest}

There is no conflict of interest.

\section{Acknowledgment}

This research was supported by a grant from the Swiss National Science Foundation (FNRS No. 2100-066872.01/1 and No. 205320-105940/1) and by the Center of Translational Biomechanics EPFL-CHUV-DAL.

\section{Appendix A. Supporting information}

Supplementary data asssociated with this article can be found in the online version at doi:10.1016/j.jbiomech.2009.11.004.

\section{References}

Aatola, S., 1989. Transmission of vibration to the wrist and comparison of frequency-response function estimators. Journal of Sound and Vibration 131, 497-507.

Blecha, L.D., Rakotomanana, L., Razafimahery, F., Terrier, A., Pioletti, D.P., 2009. Targeted mechanical properties for optimal fluid motion inside artificial bone substitutes. Journal of Orthopaedic Research 27, 1082-1087.

Bryant, J.D., David, T., Gaskell, P.H., King, P.H., Lond., G., 1989. Rheology of bovine bone marrow. Proceedings of the Institution of Mechanical Engineers, Part H: Journal of Engineering in Medicine 203, 71-75.

Caille, N., Thoumine, O., Tardy, Y., Meister, J.J., 2002. Contribution of the nucleus to the mechanical properties of endothelial cells. Journal of Biomechanics 35, 177-187.
Carpenter, P.W., Davies, C., Lucey, A.D., 2000. Hydrodynamics and compliant walls: does the dophin have a secret? Current Science 79, 758-765.

Chang, B.S., Lee, C.K., Hong, K.S., Youn, H.J., Ryu, H.S., Chung, S.S., Park, K.W., 2000. Osteoconduction at porous hydroxyapatite with various pore configurations. Biomaterials 21, 1291-1298.

Chevray, R., Mathieu, J., 1993. In: Topics in Fluid Mechanics. Cambridge University Press.

Fabry, B., Maksym, G.N., Butler, J.P., Glogauer, M., Navajas, D., Fredberg, J.J., 2001. Scaling the microrheology of living cells. Physical Review Letter 87, 148102.

Frangos, J.A., McIntire, L.V., and Eskin, S.G., 1988. Shear stress induced stimulation of mammalian cell metabolism. Biotechnology and Bioengineering 32, 1053-1060.

Gillespie, K.A., Dickey, J.P., 2003. Determination of the effectiveness of materials in attenuating high frequency shock during gait using filterbank analysis. Clinical Biomechanics 18, 50-59.

Goldstein, A.S., Juarez, T.M., Helmke, C.D., Gustin, M.C., Mikos, A.G., 2001. Effect of convection on osteoblastic cell growth and function in biodegradable polymer foam scaffolds. Biomaterials 22, 1279-1288.

Hildebrand, T., Ruegsegger, P., 1997. Quantification of Bone Microarchitecture with the Structure Model Index. Computer Methods in Biomechanics and Biomedical Engineering 1, 15-23.

Huang, Q., Goh, J.C., Hutmacher, D.W., Lee, E.H., 2002. In vivo mesenchymal cell recruitment by a scaffold loaded with transforming growth factor beta1 and the potential for in situ chondrogenesis. Tissue Engineering 8, 469-482.

Joshi, A., Guttenberg, R., Leu, M.C., Murray, S.L., 2006. Modeling of hand-arm vibration. In: Dong, R., Krajnak, K., Wirth, O., Wu, J. (Eds.), First American conference on human vibration. NIOSH, Morgantown, West Virginia, pp. $148-149$.

Kapur, S., Baylink, D.J., Lau, K.H., 2003. Fluid flow shear stress stimulates human osteoblast proliferation and differentiation through multiple interacting and competing signal transduction pathways. Bone 32, 241-251.

Karageorgiou, V., Kaplan, D., 2005. Porosity of 3D biomaterial scaffolds and osteogenesis. Biomaterials 26, 5474-5491.

Lau, K.H., Kapur, S., Kesavan, C., Baylink, D.J. 2006. Up-regulation of the Wnt, estrogen receptor, insulin-like growth factor-I, and bone morphogenetic protein pathways in $\mathrm{C} 57 \mathrm{BL} / 6 \mathrm{~J}$ osteoblasts as opposed to $\mathrm{C} 3 \mathrm{H} / \mathrm{HeJ}$ osteoblasts in part contributes to the differential anabolic response to fluid shear. Journal Biological Chemistry 281, 9576-9588.

Lu, J.X., Flautre, B., Anselme, K., Hardouin, P., Gallur, A., Descamps, M., Thierry, B., 1999. Role of interconnections in porous bioceramics on bone recolonization in vitro and in vivo. Journal of Material Science: Materials in Medicine 10, $111-120$.

Luppe, F., Conoir, J.M., Franklin, H., 2002. Scattering by a fluid cylinder in a porous medium: application to trabecular bone. Journal of the Acoustical Society of America 111, 2573-2582.

Malek, A.M., Alper, S.L., and S..Izumo. 1999. Hemodynamic shear stress and its role in artherosclerosis. In: "The Journal of the American Medical Association \%L malek99."pp. 2035-2042.

Mathieu, L.M., Mueller, T.L., Bourban, P.E., Pioletti, D.P., Muller, R., Manson, J.A., 2006. Architecture and properties of anisotropic polymer composite scaffolds for bone tissue engineering. Biomaterials 27, 905-916.

Paddan, G.S., Griffin, M.J., 1998. A review of the transmission of translational seat vibration to the head. Journal of Sound and Vibration 215, 863-882.

Panagakos, F.S., 1994. Transforming growth factor-alpha stimulates chemotaxis of osteoblasts and osteoblast-like cells in vitro. Biochemistry and Molecular Biology International 33, 643-650.

Pauw, M.T.M.V., Nulend, J.K., Bos, T.V., Burger, E.H., Everts.V., and Beertsen..W. 2000. Response of periodontal ligament fibroblasts and gingival fibroblasts to pulsating fluid flow: Nitric oxide and prostaglandin E2 release and expression of tissue non-specific alkaline phosphatase activity. In: Journal of Periodontal Research \%L vanderpauw00. pp. 335-343.

Pioletti, D.P., Rakotomanana, L.R., 2000. On the independence of time and strain effects in the stress relaxation of ligaments and tendons. Journal of Biomechanics 33, 1729-1732.

Qin, Y.X., Rubin, C.T., McLeod, K.J., 1998. Nonlinear dependence of loading intensity and cycle number in the maintenance of bone mass and morphology. Journal of Orthopaedic Research 16, 482-489.

Reich, K.M., Gay, C.V., Frangos, J.A., 1990. Fluid shear-stress as a mediator of osteoblast cyclic adenosine-monophosphate production. Journal of Cellular Physiology 143, 100-104

Rubin, C., Recker, R., Cullen, D., Ryaby, J., McCabe, J., McLeod, K., 2004. Prevention of postmenopausal bone loss by a low-magnitude, high-frequency mechanical stimuli: a clinical trial assessing compliance, efficacy, and safety. Journal Bone and Mineral Research 19, 343-351.

Sakai, K., Mohtai, M., Iwamoto, Y., 1998. Fluid shear stress increases transforming growth factor beta 1 expression in human osteoblast-like cells: modulation by cation channel blockades. Calcified Tissue International 63, 515-520.

Stewart, J.M., Karman, C., Montgomery, L.D., McLeod, K.J., 2005. Plantar vibration improves leg fluid flow in perimenopausal women. American Journal of Physiology Regulatory Integrative Comparative Physiology 288, R623-R629.

Tanaka, S.M., Li, J., Duncan, R.L., Yokota, H., Burr, D.B., Turner, C.H., 2003. Effects of broad frequency vibration on cultured osteoblasts. Journal of Biomechanics 36, $73-80$ 